\title{
Glutathione level and glutathione reductase activity in serum of coronary heart disease patients
}

\author{
Ewa Zuzak', Anna Horecka², Małgorzata Kiełczykowska², Aneta Dudek', Irena Musik², \\ Joanna Kurzepa ${ }^{3}$, Jacek Kurzepa ${ }^{2}$ \\ ${ }^{1}$ Department of Cardiology with Acute Coronary Syndromes Subunit, Queen Jadwiga Clinical Provincial Hospital No 2 in \\ Rzeszow, Poland \\ ${ }^{2}$ Department of Medical Chemistry, Medical University of Lublin, Poland \\ ${ }^{3}$ Ist Department of Medical Radiology, Medical University of Lublin, Poland
}

Zuzak E, Horecka A, Kiełczykowska M, Dudek A, Musik I, Kurzepa J, Kurzepa J. Glutathione level and glutathione reductase activity in serum of coronary heart disease patients. J Pre-Clin Clin Res. 2017; 11(2): 103-105. doi: 10.26444/jpccr/81277

\begin{abstract}
Introduction. Oxidative stress induced by the generation of excess reactive oxygen species is one of the cause of atherosclerosis finally leading to coronary heart disease (CHD). Glutathione reductase (GR), a flavoprotein antioxidant enzyme, regenerates glutathione (GSH) from its oxidized form, which is essential for scavenging of hydrogen peroxide by glutathione peroxidase. The aim of this study was to analyze the activity of GR and GSH level in serum of patients with various stages of CHD.

Materials and method. Sixty consecutive patients with diagnosis of stable angina (SA, $n=20)$, unstable angina (UA, $n=20)$ and non-fatal myocardial infarction $(\mathrm{MI}, \mathrm{n}=20)$ together with 20 healthy control subjects were enrolled. Venous blood samples were collected just before the coronary catheterization procedure (in study patients). GR activities and GSH concentration was determined using commercially available colorimetric kits.

Results. The highest GR activity and GSH serum level was noticed in UA patients. However, statistical evaluation showed that only glutathione reductase activity was significantly higher in serum obtained from myocardial infarction patients, compared to stable angina pectoris patients (19.52 $\pm 13.88 \mathrm{vs} 11.63 \pm 5.45 \mathrm{nmol} / \mathrm{min} / \mathrm{ml}$ for Ml and SA, respectively; $p<0.048$ ). There were no significant differences in GSH between the CHD patients and controls.

Conclusion. The elevated activity of glutathione reductase in serum of patients with unstable angina pectoris and myocardial infarction suggests the role of antioxidant system acute coronary syndromes.
\end{abstract}

\section{Key words}

glutathione reductase, glutathione, coronary heart disease, oxidative stress

\section{INTRODUCTION}

Coronary heart disease (CHD) is a major cause of death and disability in developed countries [1]. The main cause of CHD is coronary atherosclerosis. The first publications on this subject were made in the 1950s [2]. Oxidative stress is a state in which an imbalance between the formation and neutralization of free radicals leads to cell and tissue damage [3]. Reactive oxygen species (ROS) are a group of small reactive molecules, mostly free radicals, engaged in many physiological and pathological processes [4]. ROS are normally generated as by-products of oxygen metabolism. However, numerous environmental stressors, such as UV, ionizing radiations, pollutants, heavy metals or xenobiotics contribute to an increase in ROS production [3]. Superoxide radical is produced by the single electron reduction of the oxygen molecule. This reactive free radical is rapidly converted to hydrogen peroxide via superoxide dismutase (SOD), which is the only enzyme that scavenges superoxide [5]. Hydrogen peroxide is also a highly reactive molecule

Adress for correspondence: Jacek Kurzepa, Department of Medical Chemistry, Department of Medical Chemistry, Medical University of Lublin, Poland, Chodzki 4a, 20-093 Lublin, Poland

E-mail: jacekkurzepa@umlub.pl

Received: 5 October 2017; 18 December 2017 that is broken down into water by 2 types of enzymes, catalase (CAT) and glutathione peroxidase (GPx). GPx uses glutathione (GSH) as a co-factor which is oxidized by reaction with hydrogen peroxide [6]. Glutathione reductase (GR), a flavoprotein enzyme, regenerates GSH from its oxidized form, with NADPH as a source of reducing power [7].

All well-established cardiovascular risk factors (hypercholesterolaemia, hypertension, diabetes mellitus, smoking) leading to atherosclerosis, enhance ROS generation. In addition, oxidative modification of lipoproteins, endothelial cell activation, and macrophage infiltration occurring during atherogenesis, are facilitated by vascular oxidative stress [8]. Therefore, an understanding of the role of oxidative stress and antioxidant system in the pathogenesis of CHD appears to be a key issue of modern medicine. The aim of this study was to analyze the activity of GR and GSH level in serum of patients with various stages of CHD.

\section{MATERIALS AND METHOD}

Sixty consecutive patients with diagnosis of stable angina (SA, $\mathrm{n}=20$ ), unstable angina (UA, $\mathrm{n}=20$ ) or non-fatal myocardial infarction (MI, n=20) were enrolled. Patients were qualified according to criteria of European Society of Cardiology. SA 
was diagnosed in patients with chest pain during exertional activity that resolves with rest or sublingual administration of nitroglycerin $[9,10]$. UA was diagnosed in patients with clinical symptoms of an acute coronary syndrome without an elevation of troponin T, with or without ECG changes typical for ischemia [11]. Myocardial infarction was diagnosed as prolonged chest pain at rest with or without ST-segment elevation (STEMI or NSTEMI) [12, 13]. Twenty healthy individuals, age and gender matched to the study group, were included into the study as a control group.

$\mathrm{CHD}$ patients received treatment according to current ESC guidelines for the management of SA, UA or MI. Characteristics of the study and control patients are given in Table 1. All patients underwent coronary angiogram according to standard techniques. Patients with significant stenosis situated in a coronary artery segment were referred to percutaneous intervention (PCI). All subjects gave written consent for participation in the study. The study protocol was approved by the Bioethical Committee of the Medical University in Lublin, Poland (KE-0254/5/2017).

Table 1. Characteristics of study patients and controls

\begin{tabular}{|c|c|c|c|c|c|}
\hline & $S A(n=20)$ & UA $(n=20)$ & $\mathrm{MI}(\mathrm{n}=20)$ & $\begin{array}{l}\text { Control } \\
(n=20)\end{array}$ & P-value* \\
\hline Age (years) & $67.8 \pm 10.0$ & $69.2 \pm 10.5$ & $64.8 \pm 12.2$ & $62.4 \pm 6.7$ & \multirow{10}{*}{$p>0.05$} \\
\hline $\begin{array}{l}\text { Gender } \\
\text { (male/female) }\end{array}$ & $12 / 8$ & $17 / 3$ & $12 / 8$ & $11 / 9$ & \\
\hline BMI & $31.8 \pm 5.2$ & $29.7 \pm 3.7$ & $27.9 \pm 4.8$ & $26.1 \pm 5.8$ & \\
\hline $\begin{array}{l}\text { Active smokers } \\
(\%)\end{array}$ & $2(10 \%)$ & $3(15 \%)$ & $3(15 \%)$ & $1(5 \%)$ & \\
\hline $\begin{array}{l}\text { Total cholesterol } \\
(\mathrm{mg} / \mathrm{dl})\end{array}$ & $182.5 \pm 56.2$ & $174.1 \pm 49.9$ & $188.3 \pm 42.6$ & $171.2 \pm 31.8$ & \\
\hline LDL-C (mg/dl) & $111.3 \pm 52.4$ & $103.2 \pm 39.4$ & $112.1 \pm 37.6$ & $91.7 \pm 29.1$ & \\
\hline $\begin{array}{l}\text { Creatinine } \\
(\mathrm{mg} / \mathrm{dl})\end{array}$ & $0.95 \pm 0.20$ & $0.98 \pm 0.21$ & $0.98 \pm 0.31$ & $0.89 \pm 0.26$ & \\
\hline WBC $(103 / \mu \mathrm{l})$ & $7.42 \pm 1.71$ & $7.53 \pm 3.02$ & $9.25 \pm 3.65$ & $7.01 \pm 1.91$ & \\
\hline $\begin{array}{l}\text { Hypertension } \\
(\%)\end{array}$ & 15 (75\%) & $12(60 \%)$ & $8(40 \%)$ & 7 (35\%) & \\
\hline $\begin{array}{l}\text { Diabetes } \\
\text { melitus (\%) }\end{array}$ & 5 (25\%) & 7 (35\%) & $6(30 \%)$ & $1(5 \%)$ & \\
\hline
\end{tabular}

* P value applies t-test or $\mathrm{Chi}^{2}$, respectively.

Blood collection. Venous blood samples were collected from the median cubital vein just before the coronary catheterization procedure (in study patients). After clot forming, both blood samples were centrifuged and the obtained sera were immediately frozen and stored for further analysis.

Biochemical procedures. GR activities were measured in serum using Glutathione Reductase Assay Kit (Cayman, MI, USA) and expressed in $\mathrm{nmol} / \mathrm{min} / \mathrm{ml}$. GSH concentration was determined using BIOXYTECH ${ }^{\circledast}$ GSH-400 ${ }^{\mathrm{TM}}$ kit produced by OxisResearch ${ }^{\mathrm{TM}}$ (OXIS Health Products, Inc., Portland, OR, USA) and expressed in micromol of GSH/L. All measurements were performed according to manufacturer procedures.

Statistics. The differences between analyzed parameters in various stages of CHD were performed by using the Kruskal-Wallis test, followed by $\mathrm{p}<0.05$ Dunns' post-hoc test.
Otherwise, the Mann-Whitney test was used. The difference between selected parameters of the study and control groups were analyzed with usage of $\mathrm{Chi}^{2}$ or t-test (Tab. 1). Statistically significant values were considered when $\mathrm{p}<0.05$. GraphPad InStat v. 3.06 for Windows (GraphPad Software, San Diego, CA, USA) was applied for statistical analysis.

\section{RESULTS AND DISCUSSION}

The highest GR activity and GSH serum level was noticed in UA patients. Unfortunately, the Kruskal-Wallis test did not show statistically significant fluctuations in the analyzed parameters at various forms of CHD ( $>0.05$ for GR and GSH in SA, UA, MI and controls, Fig. 1 and 2). However, only the glutathione reductase activity seems to be significantly higher in serum obtained from myocardial infarction patients, compared to stable angina pectoris patients (19.52 \pm 13.88 vs $11.63 \pm 5.45 \mathrm{nmol} / \mathrm{min} / \mathrm{ml}$, Mann-Whitney test, $\mathrm{p}=0.048)$ (Fig. 1).

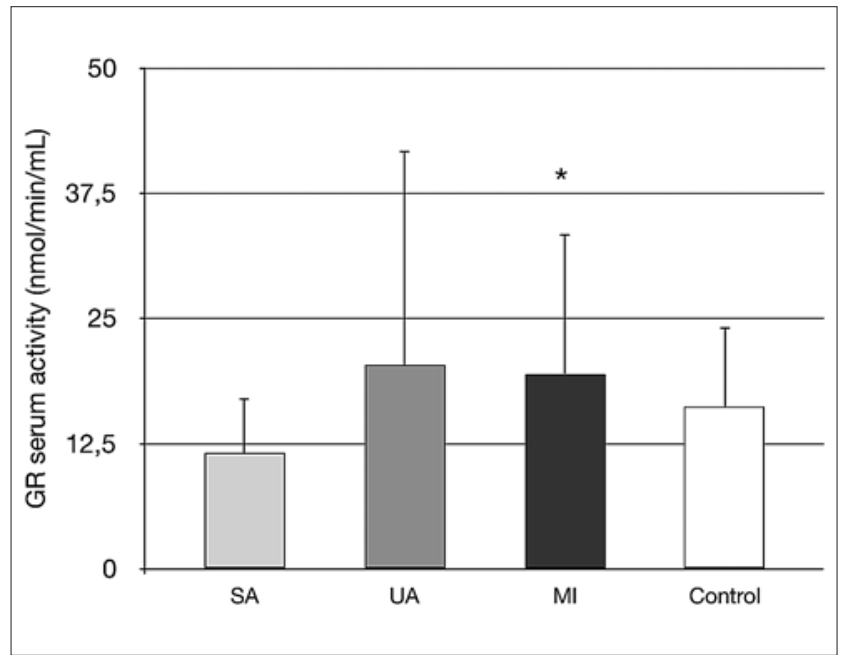

Figure 1. Glutathione reductase activity in serum patients with various stages of coronary heart disease.

$\mathrm{SA}$ - stabile angina; UA - unstable angina; $\mathrm{MI}$ - myocardial infarction. ${ }^{*} \mathrm{p}=0.048$ compared to SA patients, Mann-Whitney test.

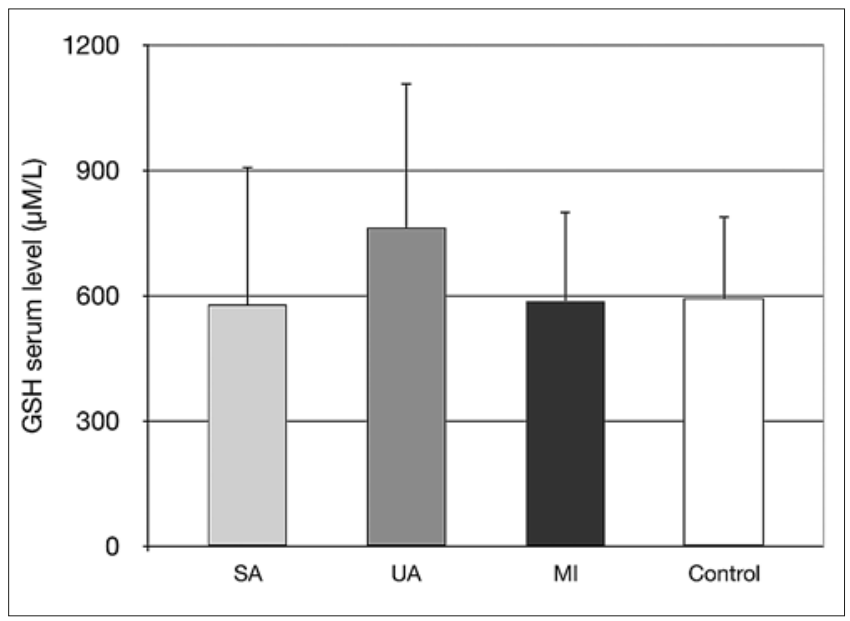

Figure 2. Reduced glutathione serum level in patients with various stages of coronary heart disease.

SA - stabile angina; UA - unstable angina; $\mathrm{MI}$ - myocardial infarction. No statistically significant differences between evaluated groups. 
Previous studies measuring GR activity and GSH level in serum indicated a decrease in both parameters in chronic lymphocytic leukemia patients [14], and significant fluctuation of GR and GSH in head and neck cancer patients during anticancer therapy [15]. However, according to the best knowledge of the authors, evaluation of both antioxidants were not performed in serum of patients simultaneously with various stages of CHD.

Kaur et al. revealed that the glutathione reductase level in platelets of unstable angina patients was markedly lowered compared to healthy subjects [16]. Such a finding indicates a decreased ability to reduce glutathione and reduced efficiency of GPx in removing hydrogen peroxide in unstable angina patients. However, higher plasmatic GR activity was observed by Sapira et al. at the admission in patients with unstable angina, compared to GR activity at discharge or to the control group [17]. In addition, GR were higher only in patients with an acute cardiovascular event, suggesting that GR activity can be considered an independent predictor of cardiovascular events during follow-up. The results of the presented study are consistent with the above mentioned findings. Unfortunately, despite the increase in GR activity, no increase in GSH concentration was observed which could be due to the rapid oxidation of GSH in the GPx reaction.

Uppal et al. noticed a significantly lower GSH level in the blood of patients with unstable angina and myocardial infarction, compared to stable angina patients and controls [18]. At the same time, the concentration of the final product of lipid peroxidation, malonyldialdehyde (MDA), was higher in UA and MI patients. It was concluded that a negative relationship between MDA and GSH may be due to the utilization of GSH during the scavenging of free radicals. However, still persisting oxidative stress in UA and MI patients led to an increase in MDA levels due to increased lipid peroxidation.

\section{CONCLUSION}

The elevated activity of glutathione reductase in the serum of patients with unstable angina pectoris and myocardial infarction suggests the role of antioxidant system acute coronary syndromes.

\section{REFERENCES}

1. Sanchis-Gomar F, Perez-Quilis C, Leischik R, Lucia A. Epidemiology of coronary heart disease and acute coronary syndrome. Ann Transl Med. 2016;4(13):256.

2. Gresham GA, Howard AN. Atherosclerosis and coronary heart-disease. Lancet. 1960;2(7149):527.

3. Pizzino G, Irrera N, Cucinotta M, Pallio G, Mannino F, Arcoraci V, et al. Oxidative Stress: Harms and Benefits for Human Health. Oxid Med Cell Longev. 2017;2017:8416763.

4. Kattoor AJ, Pothineni NVK, Palagiri D, Mehta JL. Oxidative Stress in Atherosclerosis. Curr Atheroscler Rep. 2017;19(11):42.

5. Griess B, Tom E, Domann F, Teoh-Fitzgerald M. Extracellular superoxide dismutase and its role in cancer. Free Radic Biol Med. 2017;112:464-79.

6. Wolonciej M, Milewska E, Roszkowska-Jakimiec W. Trace elements as an activator of antioxidant enzymes. Postepy Hig Med Dosw (Online). 2016;70(0):1483-98.

7. Couto N, Wood J, Barber J. The role of glutathione reductase and related enzymes on cellular redox homoeostasis network. Free Radic Biol Med. 2016;95:27-42.

8. Forstermann U, Xia N, Li H. Roles of Vascular Oxidative Stress and Nitric Oxide in the Pathogenesis of Atherosclerosis. Circ Res. 2017;120(4):713-35.

9. Tobin KJ. Stable angina pectoris: what does the current clinical evidence tell us? J Am Osteopath Assoc. 2010;110(7):364-70.

10. Montalescot G, Sechtem U, Achenbach S, Andreotti F, Arden C, Budaj A, et al. 2013 ESC guidelines on the management of stable coronary artery disease: the Task Force on the management of stable coronary artery disease of the European Society of Cardiology. Eur Heart J. 2013;34(38):2949-3003.

11. Roffi M, Patrono C, Collet JP, Mueller C, Valgimigli M, Andreotti F, et al. 2015 ESC Guidelines for the management of acute coronary syndromes in patients presenting without persistent ST-segment elevation: Task Force for the Management of Acute Coronary Syndromes in Patients Presenting without Persistent ST-Segment Elevation of the European Society of Cardiology (ESC). Eur Heart J. 2016;37(3):267-315.

12. Thygesen K, Alpert JS, Jaffe AS, Simoons ML, Chaitman BR, White HD, et al. Third universal definition of myocardial infarction. Circulation. 2012;126(16):2020-35.

13. Deckers JW. Classification of myocardial infarction and unstable angina: a re-assessment. Int J Cardiol. 2013;167(6):2387-90.

14. Bakan N, Taysi S, Yilmaz O, Bakan E, Kuskay S, Uzun N, et al. Glutathione peroxidase, glutathione reductase, $\mathrm{Cu}-\mathrm{Zn}$ superoxide dismutase activities, glutathione, nitric oxide, and malondialdehyde concentrations in serum of patients with chronic lymphocytic leukemia. Clin Chim Acta. 2003;338(1-2):143-9.

15. Patel BP, Raval GN, Rawal RM, Patel JB, Sainger RN, Patel MM, et al. Serum glutathione-S-transferase and glutathione reductase activity in head and neck cancer patients. Neoplasma. 2002;49(4):260-6.

16. Kaur G, Misra MK, Sanwal GG, Shanker K, Chandra M. Levels of glutathione reductase and glutathione peroxidase of human platelets in unstable angina and myocardial infarction. Boll Chim Farm. 1999;138(8):437-9.

17. Sapira V, Cojocaru IM, Socoliuc G, Lilios G, Grigorian M, Craiu E, et al. Glutathione reductase levels in patients with unstable angina. Rom J Intern Med. 2011;49(3):197-201.

18. Uppal N, Uppal V, Uppal P. Progression of Coronary Artery Disease (CAD) from Stable Angina (SA) Towards Myocardial Infarction (MI): Role of Oxidative Stress. J Clin Diagn Res. 2014;8(2):40-3. 